PROCEEDINGS OF THE

AMERICAN MATHEMATICAL SOCIETY

Volume 126, Number 10, October 1998, Pages 3131-3132

S $0002-9939(98) 04988-0$

\title{
A GENERALIZATION OF 2-HOMOGENEOUS CONTINUA BEING LOCALLY CONNECTED
}

\author{
KEITH WHITTINGTON
}

(Communicated by Alan Dow)

\begin{abstract}
An elementary proof is given that if each pair of points of a homogeneous metric continuum can be mapped by a homeomorphism into an arbitrarily small connected set, then the continuum is locally connected.
\end{abstract}

In [3], Ungar answered a question of Burgess [1] by showing that every 2homogeneous metric continuum is locally connected. Ungar's proof uses Theorem 2.1 of [2], a powerful result of Effros. This note gives a short, elementary proof which significantly generalizes Ungar's result without using the Effros theorem.

Lemma 1. If $X$ is a space with a countable base such that for all $x, y \in X$, there is a compact connected set $C$ containing $x$ and $y$, and an open set $U$ containing $C$ such that the component of $U$ containing $C$ is nowhere dense in $X$, then $X$ is first category.

Proof. Let $p \in X$. Since $X$ has a countable base, it follows that there is a base $U_{1}, U_{2}, \ldots$ which is closed with respect to taking finite unions. Let $V_{1}, V_{2}, \ldots$ be the elements of this list which contain $p$ and in which the component containing $p$ is nowhere dense in $X$. Let $C_{i}$ be the component of $V_{i}$ which contains $p$. We claim that the $C_{i}$ cover $X$.

Let $x \in X$. Then there is a continuum $C$ in $X$ containing $p$ and $x$, and an open set $U$ containing $C$ such that the component of $U$ containing $C$ is nowhere dense in $X$. There exist $U_{n_{1}}, U_{n_{2}}, \ldots, U_{n_{t}}$ covering $C$ such that $U_{n_{i}} \subseteq U$ for each $i$. Now $p \in U_{n_{1}} \cup U_{n_{2}} \cup \cdots \cup U_{n_{t}}$, and the component of this set containing $p$ is nowhere dense in $X$. Thus, $U_{n_{1}} \cup U_{n_{2}} \cup \cdots \cup U_{n_{t}}=V_{i}$ for some $i$, and so $x \in C_{i}$.

Lemma 2. If $X$ is a homogeneous, complete metric space that is not locally connected, then $X$ has a base of open sets, each component of which is nowhere dense in $X$.

Proof. Since $X$ is homogeneous, it suffices to show that there is a nonempty open set $V$, each component of which is nowhere dense in $X$. Suppose there is no such set. Let $U_{1}$ be some particular nonempty open set of diameter $<1$. Then $U_{1}$ has a component $C_{1}$ such that $\left(\overline{C_{1}}\right)^{\circ}$ is nonempty. There is a nonempty open set $U_{2}$ of diameter $<1 / 2$ such that $\overline{U_{2}} \subseteq\left(\overline{C_{1}}\right)^{\circ}$. Again, $U_{2}$ must have a component $C_{2}$ such $\left(\overline{C_{2}}\right)^{\circ}$ is nonempty. Continuing in this manner, one finds connected sets $\overline{C_{i}}$

Received by the editors January 30, 1998 and, in revised form, March 19, 1998.

1991 Mathematics Subject Classification. Primary 54F15.

Key words and phrases. Homogeneous, locally connected.

(C) 1998 American Mathematical Society 
that form a neighborhood base at some point $p$ in $X$. Since $X$ is homogeneous, it follows that $X$ is locally connected, contrary to the hypothesis.

Theorem 1. If $X$ is a homogeneous Polish (separable, complete metric) space such that for each pair of points $x, y \in X$ there is a point $p \in X$ such that for every $\epsilon>0$ there exists a homeomorphism $f: X \rightarrow X$ and a continuum $D$ contained in the $\epsilon$-neighborhood of $p$ such that $f(x), f(y) \in D$, then $X$ is locally connected.

Proof. Since $X$ is separable metric, it has a countable base. Suppose $X$ is not locally connected; then $X$ has a base of open sets, each component of which is nowhere dense in $X$. We will show that the remaining hypothesis of Lemma 1 is fulfilled, contradicting that $X$ is second category.

Let $x, y \in X$, and let $p$ be as above. Then there is an $\epsilon>0$ such that each component of $B(p, \epsilon)$, the $\epsilon$-ball centered at $p$, is nowhere dense in $X$. By hypothesis, there is a homeomorphism $f: X \rightarrow X$ such that $f(x)$ and $f(y)$ lie in a continuum $D$ contained in $B(p, \epsilon)$. The sets $C=f^{-1}(D)$ and $U=f^{-1}(B(p, \epsilon))$ fulfill the requirements of Lemma 1.

Theorem 2. If $X$ is a compact metric homogeneous space such that each pair of points can be mapped by homeomorphisms from $X$ to $X$ into connected sets of arbitrarily small diameter, then $X$ is locally connected.

Proof. Let $x, y \in X$. The proof is as above except that in this case if $X$ is not locally connected we can cover $X$ with finitely many open sets, each component of which is nowhere dense in $X$. Utilizing a Lebesgue number for such a cover, it easily follows from the hypothesis that there is a homeomorphism $f: X \rightarrow X$ such that $f(x)$ and $f(y)$ lie in a continuum $D$ contained in an open set $V$ in this cover. The sets $C=f^{-1}(D)$ and $U=f^{-1}(V)$ fulfill the requirements of Lemma 1.

A space $X$ is 2-homogeneous if for all points $a, b, c, d \in X$, with $a \neq b$ and $c \neq d$, there is a homeomorphism from $X$ to itself which carries the set $\{a, b\}$ into the set $\{c, d\}$. If $x$ and $y$ are distinct elements of a 2-homogeneous space, $z$ is a third point, and $f$ is a homeomorphism carrying $\{x, z\}$ into $\{y, z\}$, then either $f$ or $f^{2}$ maps $x$ to $y$; thus, every 2-homogeneous continuum is homogeneous. Since nondegenerate metric continua always have nondegenerate connected subsets of small diameter, Ungar's result immediately follows.

Corollary ([3, 3.12]). Every 2-homogeneous metric continuum is locally connected.

\section{REFERENCES}

1. C. E. Burgess, Homogeneous continua, Summary of Lectures and Seminars, Summer Institute on Set Theoretic Topology, University of Wisconsin (1955), 75-78.

2. E. G. Effros, Transformation groups and $C^{*}$-algebras, Ann. of Math. (2) 81 (1965), 38-55. MR 30:5175

3. G. S. Ungar, On all kinds of homogeneous spaces, Trans. Amer. Math. Soc. 212 (1975), 393-401. MR 52:6684

Department of Mathematics, University of the Pacific, Stockton, California 95211

E-mail address: kwhittington@uop.edu 